\title{
Four Anti-dsDNA Antibody Assays in Relation to Systemic Lupus Erythematosus Disease Specificity and Activity
}

Helena Enocsson, Christoffer Sjöwall, Lina Wirestam, Charlotte Dahle, Alf Kastbom, Johan Ronnelid, Jonas Wetterö and Thomas Skogh

The self-archived postprint version of this journal article is available at Linköping University Institutional Repository (DiVA):

http://urn.kb.se/resolve?urn=urn:nbn:se:liu:diva-118866

N.B.: When citing this work, cite the original publication.

Enocsson, H., Sjöwall, C., Wirestam, L., Dahle, C., Kastbom, A., Ronnelid, J., Wetterö, J., Skogh, T., (2015), Four Anti-dsDNA Antibody Assays in Relation to Systemic Lupus Erythematosus Disease Specificity and Activity, Journal of Rheumatology, 42(5), 817-825.

https://doi.org/10.3899/jrheum.140677

Original publication available at:

https://doi.org/10.3899/jrheum.140677

Copyright: Journal of Rheumatology

http://www.jrheum.com/ 
Four anti-dsDNA antibody assays in relation to systemic lupus erythematosus disease specificity and activity

Helena Enocsson ${ }^{1}$, Christopher Sjöwall ${ }^{1}$, Lina Wirestam ${ }^{1}$, Charlotte Dahle ${ }^{1}$, Alf Kastbom ${ }^{1}$, Johan Rönnelid ${ }^{2}$, Jonas Wetterö ${ }^{1}$, Thomas Skogh ${ }^{1}$

\section{Abstract}

Objective. Analysis of antibodies against double-stranded (ds) DNA is an important diagnostic tool for systemic lupus erythematosus (SLE), and changes in anti-dsDNA antibody levels are also used to assess disease activity. Herein, four assays were compared with regard to SLE specificity, sensitivity and association with disease activity variables.

Methods. Cross-sectional sera from 178 SLE patients, of which 11 were followed consecutively, from a regional Swedish SLE register were analyzed for IgG anti-dsDNA by bead-based multiplex assay (FIDIS, Theradig), fluoroenzyme-immunoassay (EliA, Phadia/Thermo Fisher Scientific), Crithidia luciliae immunofluorescence test (CLIFT, ImmunoConcepts) and lineblot (EUROLINE, Euroimmun). All SLE patients fulfilled the 1982 American College of Rheumatology and/or the 2012 Systemic Lupus International Collaborating Clinics (SLICC-12) classification criteria. Healthy individuals ( $n=100)$, patients with rheumatoid arthritis ( $n=95)$, and primary Sjögren's syndrome $(n=54)$ served as controls. Results. CLIFT had the highest SLE specificity (98\%), whereas EliA had the highest sensitivity (35\%). When cut-off levels for FIDIS, EliA and EUROLINE were adjusted according to SLICC-12 (i.e. twice above reference limit when using ELISA), the specificity and sensitivity of FIDIS was comparable to CLIFT. FIDIS and CLIFT also showed the highest concordance (84\%). FIDIS performed best regarding association with disease activity in cross-sectional and consecutive samples. Fisher's exact test revealed striking differences between methods regarding associations with certain disease phenotypes. 
Conclusion. CLIFT remains a good choice for diagnostic purposes, but FIDIS performs equally well when the cut-off is adjusted according to SLICC-12. Based on results from crosssectional and consecutive analyses, FIDIS can also be recommended to monitor disease activity.

Key indexing terms: Systemic lupus erythematosus, double stranded DNA, immunoassay, autoantibodies, inflammation, rheumatic disease.

Affiliations: 1) Department of Clinical and Experimental Medicine, Linköping University, Linköping, Sweden; 2) Department of Immunology, Genetics and Pathology, Uppsala University, Sweden.

Fundings: This work was supported by the Swedish Research Council K2012-69X-14594-10-3 and K2011-68X-20611-04-3]; the Swedish Society for Medicine [SLS-331171 and SLS331171]; the Swedish Society against Rheumatism [R-313701, R-307291]; the Swedish Society for Medical Research; the King Gustaf V 80-year Foundation [FAI2013-0066]; and Professor Nanna Svartz foundation.

Authors: H. Enocsson Post doc. PhD, C. Sjöwall Associate Prof. MD PhD, L. Wirestam PhDstudent MSc, C. Dahle Associate Prof. MD PhD, A. Kastbom MD PhD, J. Rönnelid Prof. MD PhD, J. Wetterö Associate Prof. PhD, T. Skogh Prof. MD PhD

Corresponding author and address for reprint requests: Helena Enocsson, AIR, Department of Clinical and Experimental Medicine, Linköping University, SE-581 85 Linköping, Sweden. Footline: Anti-dsDNA assays in SLE 


\section{Introduction}

Systemic lupus erythematosus (SLE) is a heterogeneous disease characterized by multiorgan involvement and circulating autoantibodies against a variety of antigens, most notably nuclear antigens, i.e. antinuclear antibodies (ANA) [1]. A positive ANA test by immunofluorescence (IF) microscopy remains to be a hallmark in SLE, although it is not mandatory neither according to the American College of Rheumatology (ACR) classification criteria from 1982 (ACR-82) [2] nor to the recently postulated Systemic Lupus International Collaborating Clinics (SLICC) classification criteria for SLE [3].

ANA targeting DNA were demonstrated by several independent research groups already in 1957 [4]. In 1966 one of these groups demonstrated circulating antibodies against native/double-stranded DNA (anti-dsDNA) as well as circulating DNA in SLE patients, suggesting a pathogenic connection [5]. This concept was further implicated by elution of DNA and anti-DNA antibodies from glomeruli of patients with lupus nephritis [6-8]. The advantage of measuring antibodies against strictly double-stranded DNA (in the form of circular mitochondrial DNA in kinetoplasts of Crithidia luciliae) was brought up by Aarden et al., who developed the Crithidia luciliae immunofluorescence test (CLIFT) [9]. IgG antidsDNA analyzed by CLIFT is considered fairly specific for SLE $[4,10]$, but it is also typical of autoimmune hepatitis type 1 and can be induced in patients treated with sulfasalazine or tumor necrosis factor (TNF) inhibitors [11, 12]. Anti-dsDNA antibodies may be present before onset of clinical disease and are often associated with severe manifestations, such as glomerulonephritis $[13,14]$. As measured by CLIFT, $40-80 \%$ of SLE patients have been reported to be anti-dsDNA positive over time, depending on the disease activity and severity $[4,10,15]$. Lupus disease flares are frequently associated with increasing serum levels of 
anti-dsDNA concomitantly with decreased levels of complement proteins C1q, C3 and C4, especially in lupus nephritis [16].

Enzyme-linked immunosorbent assays (ELISA) for the measurement of anti-dsDNA have repeatedly been found to have lower disease specificity compared to CLIFT [16-20]. The new SLICC-12 criteria [3] has implemented this knowledge and recommends a cut-off for ELISAs that is twice above the "laboratory reference" [3].

Many laboratories in Scandinavia (including our own) use CLIFT not only for diagnostic purposes, but also when monitoring changes in disease activity over time. Due to the relatively time consuming CLIFT-procedure as well as because of its semi quantitative nature, another rapid test with improved ability to reflect clinically relevant fluctuations in anti-dsDNA levels is highly warranted, given a specificity comparable with CLIFT. The present study was undertaken to evaluate alternative assays that could complement or replace the CLIFT. Four different anti-dsDNA detection assays were thus compared regarding disease specificity, disease sensitivity and disease activity: the CLIFT, a fluoroenzyme-immunoassay (EliA), a bead-based multiplex assay (fluorescent microsphere immunodetection system; FIDIS), and a lineblot assay (EUROLINE). In view of the new SLICC-12 criteria, diagnostic specificity and sensitivity performances of the different assays were evaluated with and without an elevated cut-off limit. Cross-sectional, as well as longitudinal, serum samples from a well-characterized Swedish SLE cohort formed the basis of this investigation. 


\section{Subjects and Methods}

\section{Patients and controls}

A total of 178 patients meeting ACR-82 and/or the 2012 SLICC classification criteria (SLICC12) were included in the study $[2,3]$. All SLE patients took part in a prospective, structured follow-up program at the rheumatology clinic, Linköping University Hospital, Sweden [21, 22]. A total of 155 patients fulfilled ACR-82 (87\%), 173 fulfilled SLICC-12 (97\%) and 150 patients fulfilled both criteria (84\%). Prevalent (91\%) as well as incident cases (9\%) were recruited consecutively during 2008-2011 and the disease duration at study inclusion ranged from 0 to 45 years with a mean of 11 years. Disease activity was recorded at every visit using the SLE disease activity index 2000 (SLEDAI-2K) [23] as well as the physician's global assessment of disease activity (PGA 0-4) [24]. The mean SLEDAI-2K was 2.3 (range 0-16), mean age at inclusion was 50 years (range 18-88), 90\% were females and 90\% were Caucasians. The mean number of met ACR criteria was 4.8 , and $21 \%$ fulfilled the ACR- 82 renal disorder criterion (other criteria are presented in Table 1). Hydroxychloroquine (HCQ) alone was prescribed to $42 \%$ of the patients, $30 \%$ were prescribed other disease modifying anti-rheumatic drugs (DMARDs) with or without $\mathrm{HCQ}$, and $65 \%$ were treated with prednisolone. The most frequently prescribed DMARDs were mycophenolate mofetil $(11 \%$ of the patients) and methotrexate (10\%) whereas other DMARDs (azathioprine, sirolimus, rituximab and ciklosporin) were less common. About half of the patients (48\%) had tested anti-dsDNA positive by CLIFT (the clinical routine method at Linköping University Hospital) at any occasion during their disease course. Eleven SLE patients representing different disease 
manifestations and with varying disease activity (SLEDAI-2K difference of $\geq 6$ ) over time were selected for consecutive anti-dsDNA analyses.

Patients with primary Sjögren's syndrome (pSS) and patients with rheumatoid arthritis (RA) served as disease controls. Serum samples were collected from 54 patients ( $96 \%$ women; mean age 62 years) meeting the American-European consensus criteria for pSS [25]. Half of the pSS patients (50\%) had a history of extraglandular disease. $89 \%$ were positive for antiSSA (with or without anti-SSB), 50\% took prednisolone, $54 \%$ were on HCQ and $29 \%$ were prescribed other DMARDs of which methotrexate was the most common (15\%).

The RA patients ( $n=95)$ were newly diagnosed ( $\leq 12$ months since first joint swelling) and included in the Swedish early RA cohort 'TIRA' between 1996 and 1998 [26]. None of the patients were on DMARD at inclusion in the study. The mean age was 55 years, $69 \%$ were women, $64 \%$ were anti-cyclic citrullinated peptide 2 (anti-CCP2) positive, and 60\% were rheumatoid factor (RF) positive. During 8 years of follow-up, none of the patients developed SLE. Control sera from 100 blood donors (50\% women; mean age 41 years) at Linköping University Hospital were also analyzed.

\section{IF-microscopy-CLIFT}

Microscope slides with fixed Crithidia luciliae (ImmunoConcepts, Sacramento, CA) were incubated for 30 min with serum diluted 1:10 in phosphate-buffered saline (pH 7.4). After washing and 30 min incubation with fluorescein-isothiocyanate (FITC-) conjugated $\gamma$-chain specific polyclonal rabbit anti-human IgG (DAKO A/S, Glostrup, Denmark), the slides were mounted in Pro-Long Gold fluorescent mounting medium (Molecular Probes-Life 
technologies, Thermofisher Scientific, MA), and evaluated by indirect IF-microscopy at 400x magnification. CLIFT-positive sera at the screening dilution $\left(>99^{\text {th }}\right.$ percentile among 100 blood donors, 50 women/50 men) were end-point titrated in 2-fold dilution steps.

\section{Lineblot -EUROLINE}

Anti-dsDNA measurement by lineblot was performed with 'ANA Profile 5 EUROLINE' test kit on a EUROBlotmaster instrument (Euroimmun AG, Lübeck, Germany). The assay, which uses native dsDNA isolated from salmon testes as antigen, was run according to manufacturer's instructions. Briefly, the immunoblot strips were incubated with a diluted serum sample (1:101) for 30 minutes. After washing, alkaline phosphatase-labelled $\gamma$-chain specific polyclonal goat anti-human IgG was added and incubated for 30 minutes. The strips were then washed and substrate solution (nitrobluetetrazoliumchlorid/5-bromo-4-chloro-3indolylphosphate) was added and incubated for 10 minutes. The reaction was stopped by washing the strips with distilled water and the test strips were evaluated with EUROLINEScan (Euroimmun AG). The blot intensity was quantified by densitometry. A signal intensity of $\geq 11$ was considered positive according to manufacturer, whereas $\geq 22$ was the cut-off used when applying the strict SLICC-12 criteria.

\section{Fluoroenzyme-immunoassay-EliA ${ }^{\text {TM }}$}

Anti-dsDNA detection by EliA ${ }^{\mathrm{TM}}$ uses recombinant circular plasmid dsDNA as antigen and was performed on the Phadia250 instrument (EliA ${ }^{\mathrm{TM}}$ dsDNA; Phadia, Uppsala, Sweden; now part of Thermo Fisher Scientific). The assay was run according to manufacturer's instructions. In short, serum samples were added to the instrument where they were diluted 1:10 and added to antigen-coated wells. After incubation and washing, monoclonal $\gamma$-chain specific 
anti-human IgG conjugated with $\beta$-galactosidase was added to the wells. Development solution ( $0.01 \%$ 4-methylumbelliferyl- $\beta$-D-galactoside) was then applied and the reaction terminated by adding a stop solution ( $4 \%$ sodium carbonate). An antibody concentration of $\geq 16 \mathrm{IU} / \mathrm{ml}$ (manufacturer's recommendation) or $\geq 32 \mathrm{IU} / \mathrm{mL}$ (SLICC-12 recommendation) was considered positive. Samples above the assay range ( $\geq 379 \mathrm{lU} / \mathrm{ml})$ were given a value of $450 \mathrm{IU} / \mathrm{mL}$.

\section{Bead-based multiplex assay with Luminex ${ }^{\circledR}-x M A P$ technology FIDIS ${ }^{\text {TM }}$}

This bead assay (Theradiag, Marne-la-Valée, Croissy Beaubourg, France) utilizes recombinant circular plasmid dsDNA as antigen. The FIDIS Connective profile test was run according to the manufacturer's instruction. Briefly, $100 \mu \mathrm{l}$ of samples diluted 1:201 were added to beads coated with 13 different ANA-associated antigens for 30 minutes in a 96well plate with filter membrane bottoms. After washing with a vacuum device, a phycoerythrin-conjugated gamma-chain specific detection antibody was added for 30 minutes. After further washing, the plate was read and evaluated using a FIDIS analyzer and the MLX-Booster software, using Luminex ${ }^{\circledR}$-xMAP technology. A concentration of $\geq 40 \mathrm{IU} / \mathrm{ml}$ was considered positive according to the manufacturer and $\geq 80 \mathrm{IU} / \mathrm{ml}$ was the cut-off when applying the SLICC-12 criteria.

\section{Other laboratory analyses}

All SLE patient visits included analysis of blood cell counts, urine erythrocytes, urine albumin, erythrocyte sedimentation rate (ESR), interferon alpha (IFN $\alpha$ ), levels of complement proteins $\mathrm{C} 3$ and $\mathrm{C} 4$ measured by nephelometry in fresh frozen plasma samples, and classic complement function assessed by a hemolytic assay. IFN $\alpha$ was measured at 
Uppsala University by a dissociation-enhanced lanthanide fluorescent immunoassay [27, 28]. Complement analyses were performed at Uppsala University Hospital or Linköping University Hospital, Sweden. In sera from RA patients, anti-CCP2 was detected by ELISA and agglutinating RF was assessed by nephelometry at Linköping University Hospital.

\section{Ethics}

Oral and written informed consent was obtained from all patients and controls. Study protocols regarding SLE and RA patients were approved by the Linköping University Ethical Review Board, Sweden (No. M75-08 and 96035, respectively) and the study protocol for pSS patients was approved by the Uppsala University Ethical Review Board, Sweden (No. 2006/217/2).

\section{Statistics}

Concordance between methods was defined as the sum of double positive samples and double negative samples, divided by the total number of samples, multiplied by 100 . Since values obtained by CLIFT are ordinal and EUROLINE is considered qualitative according to the manufacturer, continuous variables were tested with non-parametric tests (Spearman's correlation, Mann-Whitney's U test and Wilcoxon's Signed Rank Test) to accomplish comparability between all anti-dsDNA measurement methods. Fisher's exact test was used for categorical data. Receiver operator characteristics (ROC) were used to calculate cut-off limits for a given specificity. Two-tailed $p$-values of $<0.05$ were considered significant. Statistical analyses were performed with SPSS Statistics 21 (IBM, Armonk, NY) or GraphPad Prism 5, version 5.04 (GraphPad Software, La Jolla, CA). 


\section{Results}

\section{SLE specificity}

Serum anti-dsDNA antibodies were determined in patients and healthy controls, and the specificity and sensitivity for SLE was calculated for each of the four methods using the cutoff according to the manufacturer (FIDIS, EliA and EUROLINE) or laboratory reference (CLIFT) (Fig. 1). The highest percentage of anti-dsDNA positive SLE patients (i.e. sensitivity) was found using EliA (35\%), whereas CLIFT had the lowest sensitivity (24\%). In contrast, CLIFT displayed the highest disease specificity for SLE (98\%). FIDIS had the lowest SLE specificity, but in contrast to EliA and EUROLINE, it did not capture any healthy individuals. Increasing the cut-off for EUROLINE, FIDIS and EliA to twice above manufacturer's reference (according to recommendations in the SLICC-12 criteria for the use of ELISAs) (Fig. 1B-D), resulted in excellent specificity for all methods (98-99\%), but also reduced the sensitivity below the sensitivity for CLIFT. A ROC curve analysis rendered the best area under the curve (AUC) for EliA (0.712), followed by EUROLINE (0.621), CLIFT (0.614) and FIDIS (0.571), but adjusting the cut-off limits for FIDIS, EliA and EUROLINE to achieve an acceptable specificity (i.e. comparable to CLIFT; 98.4\%), rendered the highest sensitivity for FIDIS (24\%, cut-off at 77 $\mathrm{IU} / \mathrm{mL}$ ) followed by EliA (19\%, cut-off at $38 \mathrm{IU} / \mathrm{mL}$ ) and EUROLINE (19\%, cut-off at a signal intensity of 19).

\section{Concurrence between methods}

Correlations and concordances between methods were evaluated in the 178 cross-sectional SLE sera. Samples below the cut-off were given a value of zero to avoid influence of level 
differences among double negative samples in the correlation analyses. All methods correlated significantly with each other and had a concordance of $\geq 72 \%$ (Fig. 2). The strongest correlation and concordance at manufacturers' cut-off was found between CLIFT and FIDIS (Fig. 2A; rho $=0.623, p<0.0001$, concordance $=84 \%$ ) whereas FIDIS and EliA had the highest correlation and concordance at the increased cut-off (numbers in parenthesis in Fig. 2B; rho=0.641, $\mathrm{p}<0.0001$, concordance $=87 \%)$. EUROLINE versus EliA, and EUROLINE versus FIDIS, displayed the weakest correlations as well as the lowest concordances (Fig. 2E and 2F). When applying increased cut-off levels for FIDIS, EliA and EUROLINE, all concordances increased (concordances in parenthesis in Fig. 2A-F) except from the one between EliA and FIDIS which remained at $84 \%$ (Fig. 2A) and between EUROLINE and CLIFT which was lowered (Fig. 2C).

The overlap between methods (positive by $\geq 2$ methods at manufacturer's cut-off) in the disease control groups were $32 \%$ for pSS and $11 \%$ for RA with the highest overlap between CLIFT and FIDIS. Anti-dsDNA positive pSS patients (measured by any method) did not differ significantly from anti-dsDNA negative pSS patients with regard to the presence of one or more extraglandular manifestations. RA patients judged anti-dsDNA positive with FIDIS (manufacturer's cut-off), were more often positive also for RF (9 out of 10 patients, $p=0.047)$ and anti-CCP2 $(10 / 10, p=0.013)$ than those testing anti-dsDNA negative with FIDIS. In contrast, only 2 of 5 anti-dsDNA positive RA patients as assessed by EliA and EUROLINE tested RF-positive ( $p=0.39)$. Furthermore, RA patients that were anti-dsDNA positive by EliA (manufacturer's cut-off) tended to be anti-CCP negative $(4 / 5, p=0.054)$. 


\section{Association of anti-dsDNA with disease phenotype}

To evaluate whether any of the four anti-dsDNA assays capture specific disease phenotypes, we compared their association with individual and grouped ACR-82 criteria (Table 1). Patients meeting any skin criteria (ACR criteria 1 and/or 2 and/or 3) were less likely to be anti-dsDNA positive by CLIFT. EliA was the only assay where anti-dsDNA was associated with oral ulcers, whereas FIDIS was the only method where anti-dsDNA was related to hematological disorder. Patients fulfilling ACR criterion 7 (renal disorder) were more likely to be anti-dsDNA positive by EliA and FIDIS. The ACR 10 criterion (immunological disorder), which amongst other autoantibodies includes anti-dsDNA (herein defined by CLIFT), was associated with anti-dsDNA positivity in all assays (not shown). There were no statistically significant associations between use of specific DMARDs and anti-dsDNA positivity by any of the methods (not shown). Manufacturer's cut-off (EliA, FIDIS and EUROLINE) and laboratory reference cut-off (CLIFT) were used in these analyses.

\section{Correlation with disease variables (cross-sectional analysis)}

To evaluate the association of anti-dsDNA antibody levels with disease activity measures (SLEDAI-2K, PGA, classical complement function, ESR, and circulating levels of $\mathrm{C} 3, \mathrm{C} 4$, and IFNa) we compared the four methods in SLE patients (Table 2). The strongest correlations were found between anti-dsDNA and complement proteins and classical complement function. Anti-dsDNA assessed by FIDIS versus classical complement function showed the highest Spearman's rank correlation coefficient ( $r$ o $=-0.552, p<0.0005$ ). Overall, FIDIS displayed a higher correlation coefficient to the activity variables (Table 2). 


\section{Individual variations in anti-dsDNA}

To evaluate if anti-dsDNA levels reflect disease activity over time at an individual level we compared anti-dsDNA levels between the occasion with highest and lowest disease activity, respectively, in 11 patients that were followed consecutively. The number of patients with a $>25 \%$ increase in anti-dsDNA level (with the highest anti-dsDNA level above manufacturer's cut-off for positivity) were calculated for each method. FIDIS and EliA captured the increase in disease activity in 7 of the patients (only 5 patients for EliA when the increased cut-off was applied) while CLIFT captured 5 patients and EUROLINE detected raised disease activity in 2 of the patients. Of the 11 consecutively followed patients, 9 were anti-dsDNA positive by any method and at any visit (manufacturers' cut-off). Graphs showing anti-dsDNA levels and disease activity over time for each of these 9 patients are shown in Figure 3 A-I. Our interpretation of the graphs is that FIDIS is superior or equal to any other method in 6 patients (panel A-F), whereas EliA performs better or similarly compared to any other method in 2 patients (panel A and B) and CLIFT is superior or equal to any other method in 3 patients (panel E-G). Disease flares of the patients represented by panel $\mathrm{H}$ and I, respectively were not mirrored by anti-dsDNA by any of the methods according to our judgment. The visual interpretation was made based on how well the disease flare(s) (as denoted by SLEDAI and/or PGA) was reflected by an increase in anti-dsDNA level. EUROLINE is not included in the graphs due to its poor performance of reflecting disease activity variables in cross sectional samples.

\section{Discussion}

Based on the results of the present study, we conclude that there is a great variability between anti-dsDNA assays and that a stricter cut-off limit must be applied to achieve an 
acceptable SLE specificity of FIDIS, EliA and EUROLINE. Further, we found that FIDIS is superior to the other methods in monitoring of disease activity, but also has a specificity and sensitivity comparable to CLIFT when a strict cut-off limit is applied.

Most Swedish clinical immunology laboratories still use CLIFT for primary (or confirmatory) analysis of anti-dsDNA antibodies. At our laboratory, we routinely perform IgG-specific CLIFT in all IgG-ANA positive serum samples, regardless of IF-staining pattern. The use of a $\gamma$-chain specific secondary antibody avoids detection of low-avidity IgM-class antibodies without clinical interest [29]. High-affinity IgA and/or IgM anti-dsDNA, which may be clinically relevant $[30,31]$, were not considered in this study.

The relatively low SLE sensitivity of all assays in this study is likely due to the relatively low mean disease activity and low percentage of renal involvement (ACR criterion 7) among the SLE patients. One plausible explanation for the low prevalence of renal involvement is the ethnicity which is linked to the risk of nephritis with a lower frequency of renal involvement in white populations [32], and the fact that the study cohort includes the majority of SLE patients in the region, meaning that the whole spectrum of SLE is present including uncomplicated cases. The lowest sensitivity among the compared assays was found for CLIFT (manufacturer's cut-off/laboratory reference). The fact that CLIFT, compared to other methods, has repeatedly demonstrated a low sensitivity but high diagnostic specificity [20, $33,34]$ was considered in the formation of the SLICC-12 classification criteria for lupus [3]. In these criteria, a cut-off limit twice above laboratory reference is required when ELISA (without further specification) is used for anti-dsDNA quantification. However, the SLICC-12 criteria does not state whether this also applies for other "non-classical" assays (i.e. other 
than Farr and CLIFT) and if the laboratory reference denotes manufacturers' recommendations for cut-off, or to a cut-off determined by the clinical laboratory. In our study, an increased cut-off limit (twice above manufacturer's recommendation) indeed increased the specificity to achieve comparability with CLIFT, but at the same time, the sensitivity became very low for EUROLINE thus also introducing a risk to miss out true antidsDNA positive patients. The importance of a proper threshold, and the need for an increased cut-off limit in order to accomplish clinical usefulness for FIDIS and ELISA has also been demonstrated by others [35]. At the manufacturer's cut-off levels, FIDIS, EliA and EUROLINE all captured higher numbers of anti-dsDNA positive RA and pSS cases compared to CLIFT, and with regard to this, our results are not entirely in line with a previous study showing a specificity of EliA that was comparable to CLIFT [34].

While, in our study, the anti-dsDNA levels recorded with CLIFT showed fair correlations and reasonable concordance rates versus FIDIS (rho $=0.62 ; 84 \%$ concordance) and EUROLINE (rho=0.56; 81\%), but somewhat lower for EliA (rho=0.48; 76\%), quantification of anti-dsDNA with CLIFT and EUROLINE performed poorly regarding the ability to reflect increases in disease activity among SLE patients followed over time. We also compared the 178 crosssectional SLE sera regarding correlation with disease activity measures, and here we concluded that, apart from EUROLINE, significant positive correlations were seen between anti-dsDNA levels and some disease activity variables. However, this was not captured by the SLEDAI-2K, nor by the PGA. FIDIS displayed the highest correlation with almost all disease activity variables investigated and was also superior in reflecting disease activity over time as determined by the number of patients with an increase in anti-dsDNA level 
above $25 \%$ between lowest and highest disease activity, and visual interpretation of individual patients over time.

A clear difference between the assays was demonstrated in the negligible overlap of antidsDNA positive disease controls. This could indicate discrepancies in cross-reactivity among the assays, and also suggests that the disease controls were true non-SLE patients. In contrast to EUROLINE and EliA, FIDIS did not capture any of the healthy controls, and the low disease specificity at manufacturer's cut-off was thus due to a high number of antidsDNA positive RA and pSS patients. This could speculatively indicate an interference of the antigen with other autoantibodies, since RA and pSS are both autoimmune diseases.

The differences between assays also became apparent in the relation between anti-dsDNA and disease phenotypes defined as ACR-82 criteria. In patients with photosensitivity, there was a decreased risk of anti-dsDNA positivity by CLIFT and FIDIS only, a risk that became even more pronounced for CLIFT when summarizing the skin criteria (ACR criteria 1, 2 or 3). Surprisingly, renal disorder was not significantly associated with CLIFT, but only with FIDIS and EliA. Although the fulfilled ACR criteria only indicate the general disease phenotype, and not necessarily manifestations at blood sampling, these results reflect that anti-dsDNA antibodies are heterogeneous and that they are captured differently depending on assay. The nature of the antigen and its immobilization, as well as other experimental details e.g. buffers and incubation times are example of factors that can affect detection of autoantibodies. The importance of antigen source and immobilization can be demonstrated by the observation that EliA and FIDIS displayed the highest correlation and concordance (at increased cut-off) of the methods evaluated in this study. Both of these assays use 
recombinant circular plasmid dsDNA as antigen, in contrast to CLIFT and EUROLINE. The differences seen between EliA and FIDIS (i.e. phenotype specificity) is therefore more likely explained by other factors. One confounding factor could be interfering heterophilic antibodies and autoantibodies, most notably RF which can disturb an assay by its binding to Fc-parts of other autoantibodies. Such interference could possibly explain the differences between FIDIS and EliA since they differed vastly in their detection of RA patients, with antidsDNA detected by FIDIS being significantly associated with anti-CCP as well as RF at manufacturer's cut-off, whereas EliA was not.

To conclude, we found CLIFT and FIDIS to be the best assays for diagnostic purposes given the adjusted cut-off for FIDIS. Based on our findings, FIDIS can also be recommended to monitor disease activity, and could thus potentially replace CLIFT if one method for both diagnostics and monitoring of disease activity is preferred. Although the SLICC-12 classification criteria improves the usefulness of ELISA for the determination of anti-dsDNA by its demand for an increased cut-off, an assay-independent recommendation for cut-off limits (i.e. a cut-off based on anti-dsDNA levels in a reference population) would make these criteria even more useful.

\section{Acknowledgements}

The authors wish to thank Per Eriksson and Marianne Peterson at Linköping University Hospital for pSS patient characterization and biobank handling, respectively. Ann-Marie Fornander, Käthy Birgersson, Carina Bergvall and Christina Olsson at Linköping University Hospital and Azita Sohrabian at Uppsala University are acknowledged for help with the anti- 
H. Enocsson, C. Sjöwall, L. Wirestam, C. Dahle, A. Kastbom, J. Rönnelid, J. Wetterö, T. Skogh

dsDNA assays. Maija-Leena Eloranta, Lars Rönnblom and Anne Trönnberg at Uppsala University are acknowledged for IFN $\alpha$ measurements. 


\section{References}

1. Rahman A, Isenberg DA. Systemic lupus erythematosus. N Engl J Med 2008;358:92939.

2. Tan EM, Cohen AS, Fries JF, Masi AT, McShane DJ, Rothfield NF et al. The 1982 revised criteria for the classification of systemic lupus erythematosus. Arthritis Rheum 1982;25:1271-7.

3. Petri M, Orbai AM, Alarcon GS, Gordon C, Merrill JT, Fortin PR et al. Derivation and validation of the Systemic Lupus International Collaborating Clinics classification criteria for systemic lupus erythematosus. Arthritis Rheum 2012;64:2677-86.

4. Isenberg DA, Manson JJ, Ehrenstein MR, Rahman A. Fifty years of anti-ds DNA antibodies: are we approaching journey's end? Rheumatology (Oxford) 2007;46:1052-6.

5. Tan EM, Schur PH, Carr RI, Kunkel HG. Deoxybonucleic acid (DNA) and antibodies to DNA in the serum of patients with systemic lupus erythematosus. J Clin Invest $1966 ; 45: 1732-40$.

6. Krishnan C, Kaplan MH. Immunopathologic studies of systemic lupus erythematosus. II. Antinuclear reaction of gamma-globulin eluted from homogenates and isolated glomeruli of kidneys from patients with lupus nephritis. J Clin Invest 1967;46:569-79.

7. Koffler D, Schur PH, Kunkel HG. Immunological studies concerning the nephritis of systemic lupus erythematosus. J Exp Med 1967;126:607-24.

8. Vlahakos DV, Foster MH, Adams S, Katz M, Ucci AA, Barrett KJ et al. Anti-DNA antibodies form immune deposits at distinct glomerular and vascular sites. Kidney Int 1992;41:1690-700. 
9. Aarden LA, de Groot ER, Feltkamp TE. Immunology of DNA. III. Crithidia luciliae, a simple substrate for the determination of anti-dsDNA with the immunofluorescence technique. Ann N Y Acad Sci 1975;254:505-15.

10. Kavanaugh AF, Solomon DH, American College of Rheumatology Ad Hoc Committee on Immunologic Testing G. Guidelines for immunologic laboratory testing in the rheumatic diseases: anti-DNA antibody tests. Arthritis Rheum 2002;47:546-55.

11. Irving KS, Sen D, Tahir H, Pilkington C, Isenberg DA. A comparison of autoimmune liver disease in juvenile and adult populations with systemic lupus erythematosus-a retrospective review of cases. Rheumatology (Oxford) 2007;46:1171-3.

12. Czaja AJ, Cassani F, Cataleta M, Valentini P, Bianchi FB. Frequency and significance of antibodies to actin in type 1 autoimmune hepatitis. Hepatology 1996;24:1068-73.

13. Eriksson C, Kokkonen H, Johansson M, Hallmans G, Wadell G, Rantapaa-Dahlqvist S. Autoantibodies predate the onset of systemic lupus erythematosus in northern Sweden. Arthritis Res Ther 2011;13:R30.

14. Winfield JB, Faiferman I, Koffler D. Avidity of anti-DNA antibodies in serum and IgG glomerular eluates from patients with systemic lupus erythematosus. Association of high avidity antinative DNA antibody with glomerulonephritis. J Clin Invest 1977;59:90-6.

15. Cervera R, Khamashta MA, Font J, Sebastiani GD, Gil A, Lavilla P et al. Systemic lupus erythematosus: clinical and immunologic patterns of disease expression in a cohort of 1,000 patients. The European Working Party on Systemic Lupus Erythematosus. Medicine (Baltimore) 1993;72:113-24. 
16. Julkunen $\mathrm{H}$, Ekblom-Kullberg S, Miettinen A. Nonrenal and renal activity of systemic lupus erythematosus: a comparison of two anti-C1q and five anti-dsDNA assays and complement C3 and C4. Rheumatol Int 2012;32:2445-51.

17. Haugbro K, Nossent JC, Winkler T, Figenschau Y, Rekvig OP. Anti-dsDNA antibodies and disease classification in antinuclear antibody positive patients: the role of analytical diversity. Ann Rheum Dis 2004;63:386-94.

18. Launay D, Schmidt J, Lepers S, Mirault T, Lambert M, Kyndt X et al. Comparison of the Farr radioimmunoassay, 3 commercial enzyme immunoassays and Crithidia luciliae immunofluorescence test for diagnosis and activity assessment of systemic lupus erythematosus. Clin Chim Acta 2010;411:959-64.

19. Eaton RB, Schnneider G, Schur PH. Enzyme immunoassay for antibodies to native DNA. Specificity and quality of antibodies. Arthritis Rheum 1983;26:52-62.

20. Isenberg DA, Dudeney C, Williams W, Addison I, Charles S, Clarke J et al. Measurement of anti-DNA antibodies: a reappraisal using five different methods. Ann Rheum Dis 1987;46:448-56.

21. Enocsson H, Wetterö J, Skogh T, Sjöwall C. Soluble urokinase plasminogen activator receptor levels reflect organ damage in systemic lupus erythematosus. Transl Res 2013;162:287-96.

22. Frodlund M, Dahlström O, Kastbom A, Skogh T, Sjöwall C. Associations between antinuclear antibody staining patterns and clinical features of systemic lupus erythematosus: analysis of a regional Swedish register. BMJ Open 2013;3:e003608.

23. Gladman DD, Ibanez D, Urowitz MB. Systemic lupus erythematosus disease activity index 2000. J Rheumatol 2002;29:288-91. 
24. Scott DL. A simple index to assess disease activity in rheumatoid arthritis. J Rheumatol 1993;20:582-4.

25. Vitali C, Bombardieri S, Jonsson R, Moutsopoulos HM, Alexander EL, Carsons SE et al. Classification criteria for Sjogren's syndrome: a revised version of the European criteria proposed by the American-European Consensus Group. Ann Rheum Dis 2002;61:554-8.

26. Kastbom A, Strandberg G, Lindroos A, Skogh T. Anti-CCP antibody test predicts the disease course during 3 years in early rheumatoid arthritis (the Swedish TIRA project). Ann Rheum Dis 2004;63:1085-9.

27. Enocsson H, Sjöwall C, Skogh T, Eloranta ML, Rönnblom L, Wetterö J. Interferonalpha mediates suppression of C-reactive protein: Explanation for muted C-reactive protein response in lupus flares? Arthritis Rheum 2009;60:3755-60.

28. Cederblad B, Blomberg S, Vallin H, Perers A, Alm GV, Rönnblom L. Patients with systemic lupus erythematosus have reduced numbers of circulating natural interferon-alpha-producing cells. J Autoimmun 1998;11:465-70.

29. Bootsma H, Spronk PE, Hummel EJ, de Boer G, ter Borg EJ, Limburg PC et al. Antidouble stranded DNA antibodies in systemic lupus erythematosus: detection and clinical relevance of IgM-class antibodies. Scand J Rheumatol 1996;25:352-9.

30. Förger F, Matthias T, Oppermann M, Becker H, Helmke K. Clinical significance of antidsDNA antibody isotypes: IgG/IgM ratio of anti-dsDNA antibodies as a prognostic marker for lupus nephritis. Lupus 2004;13:36-44.

31. Villalta D, Bizzaro N, Bassi N, Zen M, Gatto M, Ghirardello A et al. Anti-dsDNA antibody isotypes in systemic lupus erythematosus: IgA in addition to IgG anti-dsDNA help to identify glomerulonephritis and active disease. PLoS One 2013;8:e71458. 
32. Hopkinson ND, Jenkinson C, Muir KR, Doherty M, Powell RJ. Racial group, socioeconomic status, and the development of persistent proteinuria in systemic lupus erythematosus. Ann Rheum Dis 2000;59:116-9.

33. Hernando M, Gonzalez C, Sanchez A, Guevara P, Navajo JA, Papisch W et al. Clinical evaluation of a new automated anti-dsDNA fluorescent immunoassay. Clin Chem Lab Med 2002;40:1056-60.

34. Antico A, Platzgummer S, Bassetti D, Bizzaro N, Tozzoli R, Villalta D et al. Diagnosing systemic lupus erythematosus: new-generation immunoassays for measurement of anti-dsDNA antibodies are an effective alternative to the Farr technique and the Crithidia luciliae immunofluorescence test. Lupus 2010;19:906-12.

35. Martin J, Durant C, Rimbert M, Hemont C, Hamidou M, Audrain M. Evaluation of two antibodies against double-stranded DNA assays in discriminating between active and non-active systemic lupus erythematosus: correlation between the cut-off and the specificity. Pathol Biol (Paris) 2012;60:387-91. 


\section{Figure legends}

Figure 1. Anti-dsDNA in SLE patients and control groups, as measured by CLIFT (A), FIDIS (B), EliA (C) and EUROLINE (D). SLE disease specificity of different anti-dsDNA antibody assays was calculated in relation to control groups (rheumatoid arthritis, primary Sjögren's syndrome and healthy individuals). The dashed line indicates cut-off for positivity according to laboratory reference $(A)$, manufacturer's recommendation (lower line in panel B-D) or twice above manufacturers' reference (upper line in panel B-D) Specificity and sensitivity for the respective cut-off limits are denoted in the tables above the graphs. 


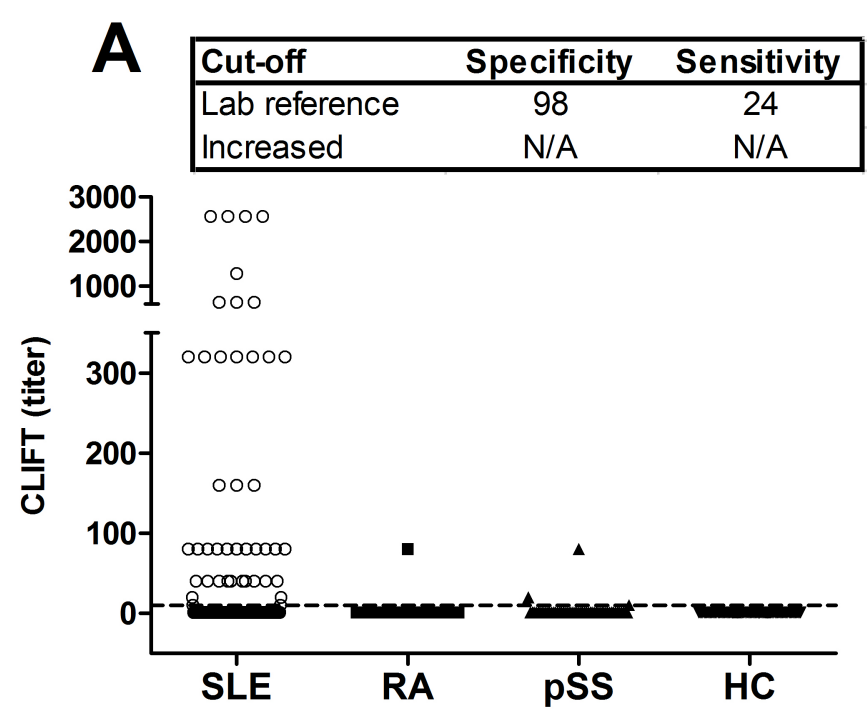

\begin{tabular}{|lcc|}
\hline Cut-off & Specificity & Sensitivity \\
\hline Manufacturer's & 92 & 30 \\
Increased & 98 & 23 \\
\hline
\end{tabular}
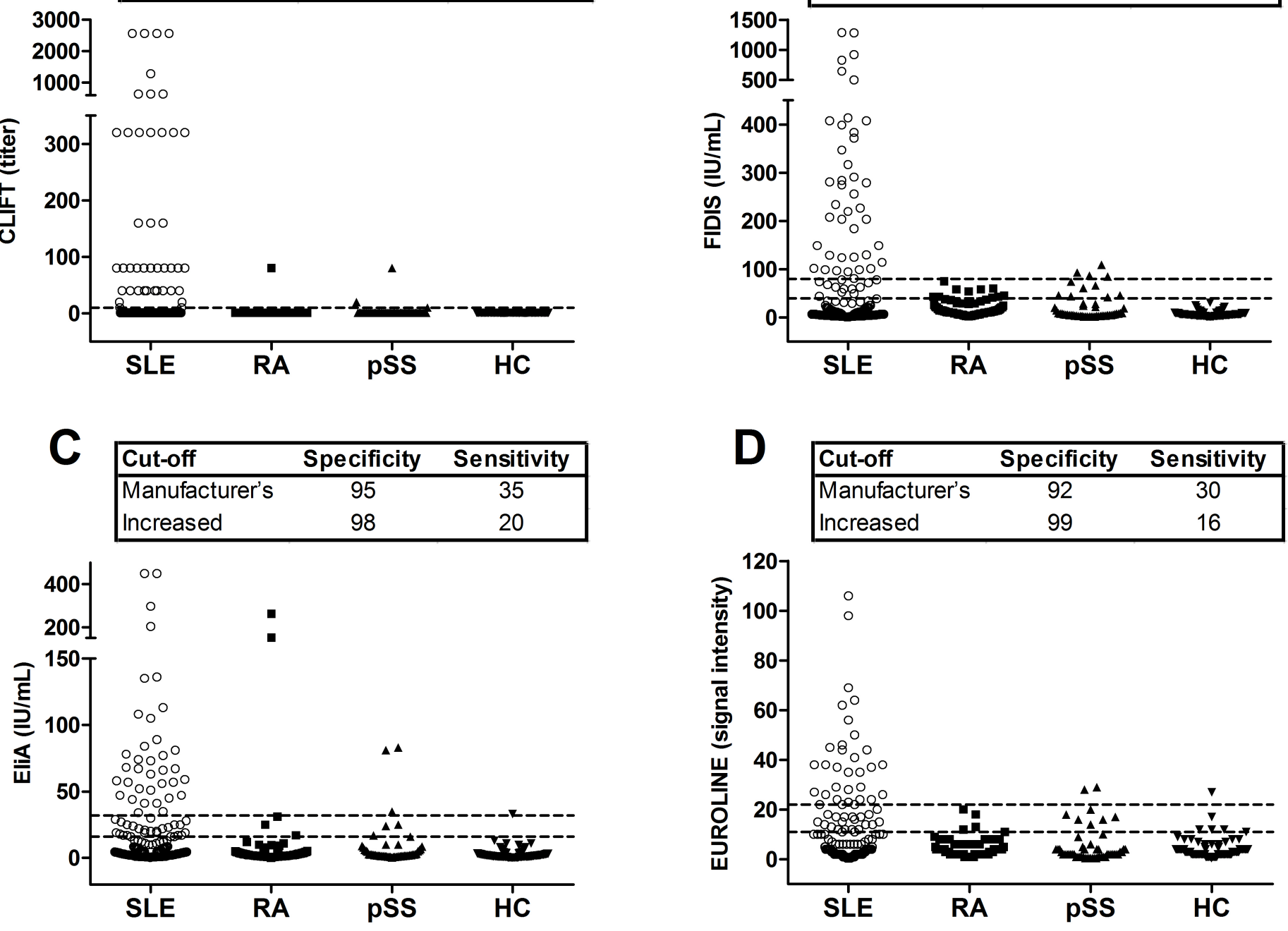
Figure 2. Correlation and concordance of anti-dsDNA assays in cross-sectional SLE samples. Panels (A-E) are sorted according to correlation. Please observe that certain dots represent more than one patient. Zero values are replaced with 1 for CLIFT and 0.5 for EUROLINE to be contained in the log-scale of the axes. Dashed lines indicate the manufacturer's cut-off for positivity (EliA, FIDIS and EUROLINE) or laboratory reference (CLIFT) and numbers designate the sum of patients in the specific quadrant. P- and rho values are from Spearman's correlation. Values below cut-off were given a value of zero to avoid unwanted influence of double negative samples in the correlation analyses. The rho values and assay concordances when adopting a cut-off limit twice above the manufacturer's recommendation are given in parenthesis. Concordance is the sum of double positive samples and double negative samples, divided by the total number of patients $(n=178)$. 
A

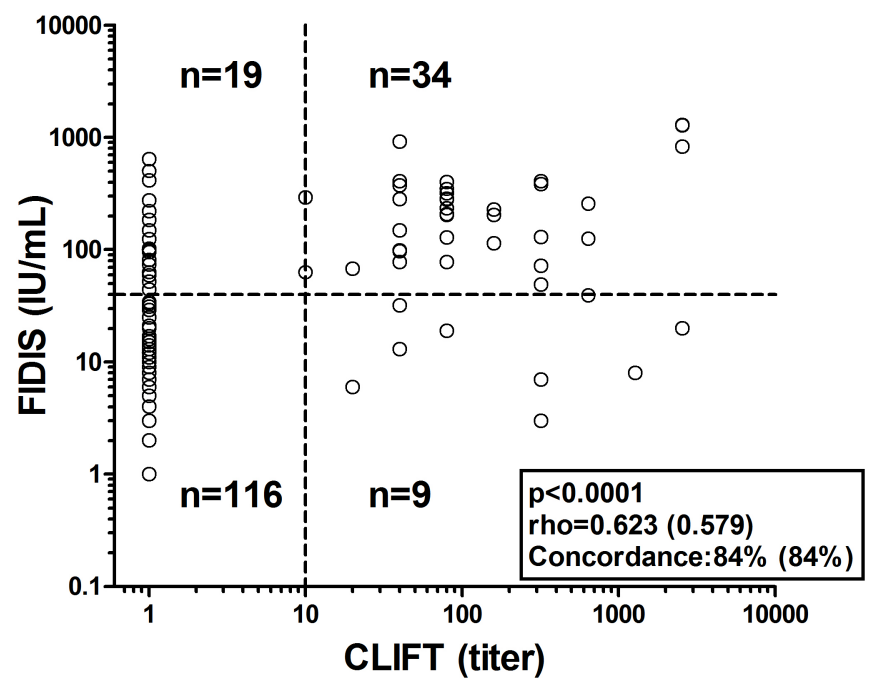

C

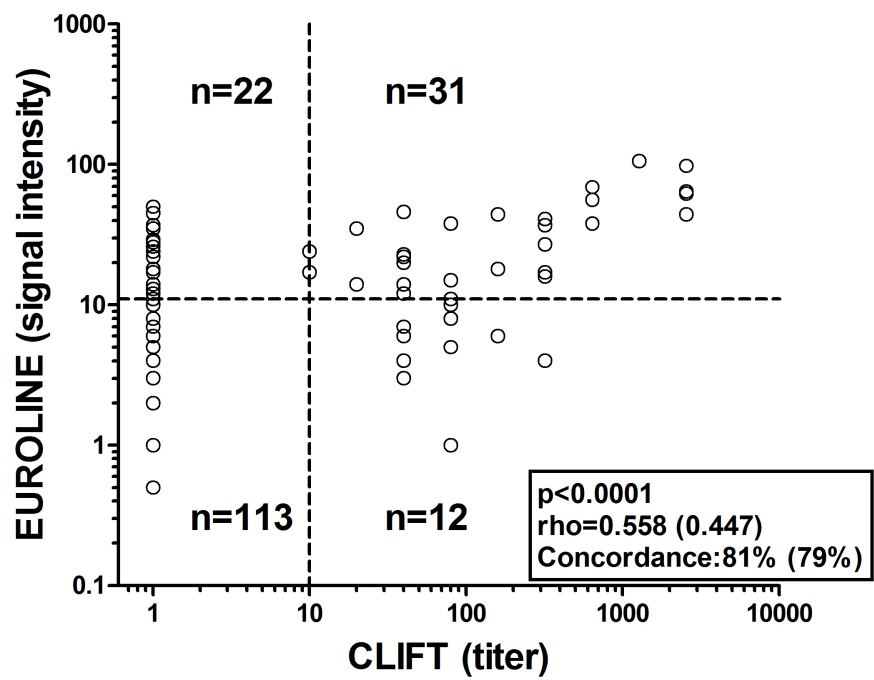

E

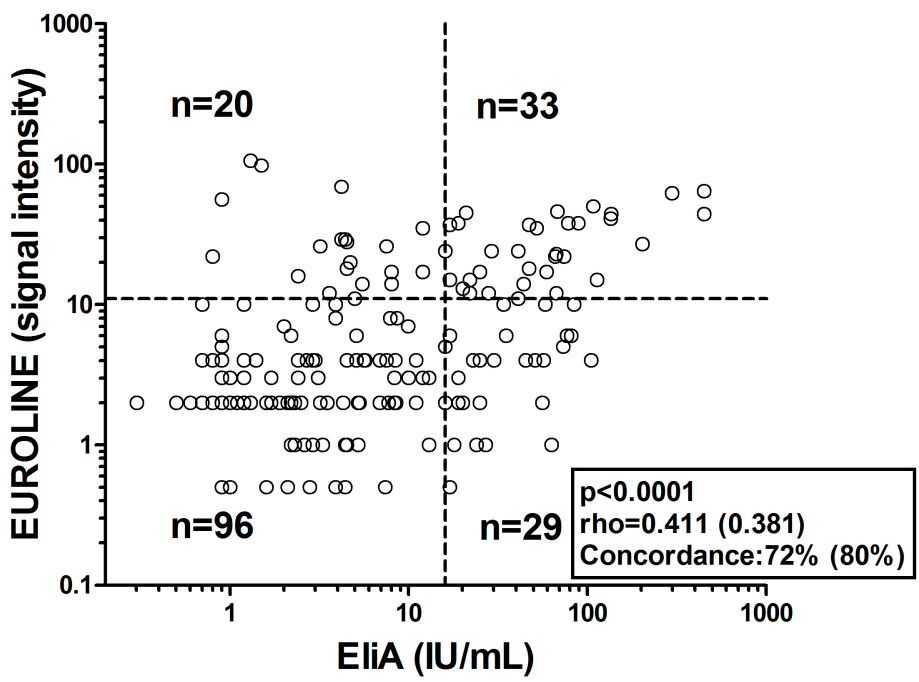

B

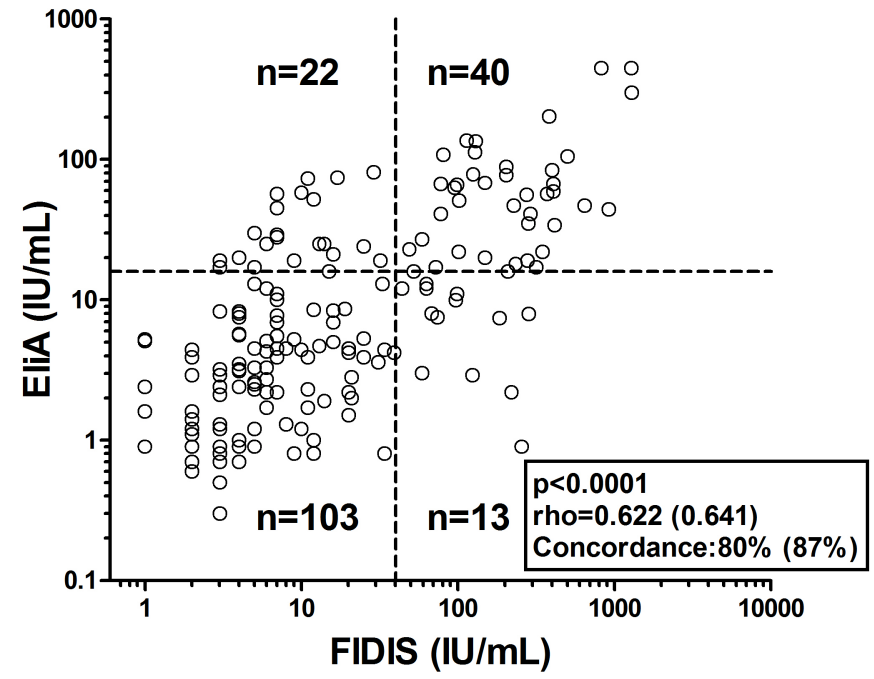

D

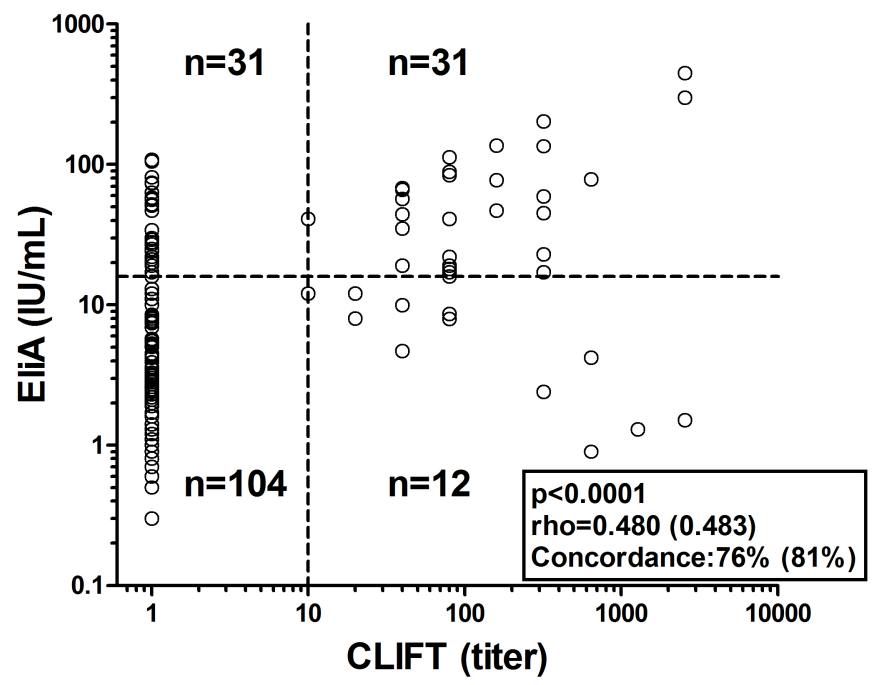

F

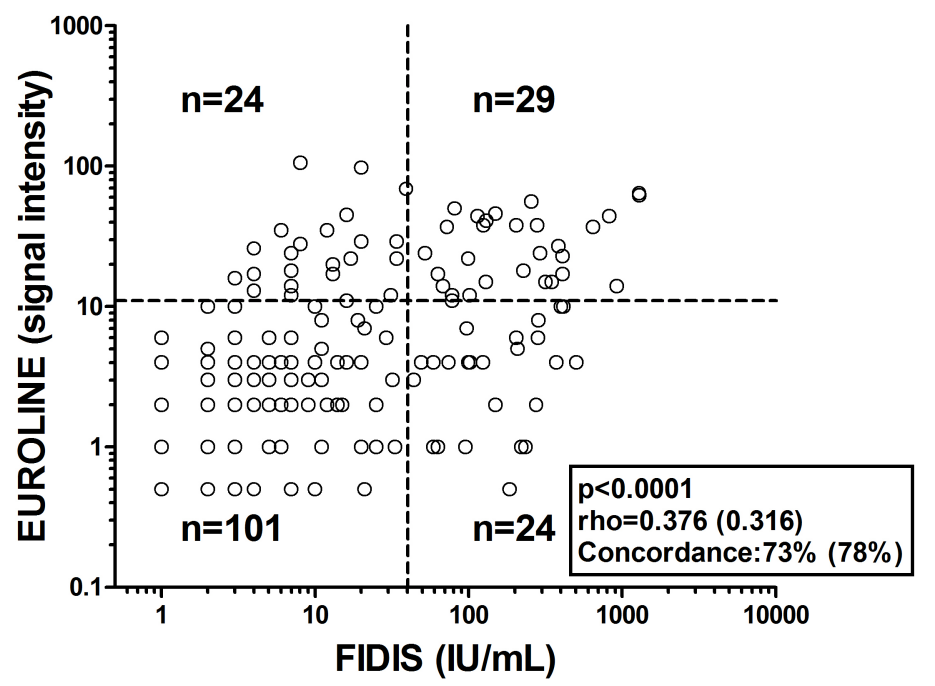


Figure 3. The graphs illustrate disease activity (PGA and SLEDAI-2K*) manifestations at present at flare, and anti-dsDNA levels in 9 patients (A-I) that were followed consecutively. Description of axes and lines are found in panel A. Observe that axes scales are different in the graphs. Disease flare manifestations of patients with several flares were:

B) Serositis and arthritis at first flare. Serositis at second flare.

E) Rash, nephritis and fever at first flare. Rash, nephritis and vasculitis at second flare.

F) Serositis and leukopenia at first flare. Nephritis at second flare.

G) Serositis and nephritis at first flare. Nephritis at second flare.

H) Epilepsy and serositis at first flare. Arthritis, rash, and epilepsy at second flare. Rash, alopecia and epilepsy at third flare. Rash, Alopecia, oral ulcers and epilepsy at fourth flare.

*item for anti-dsDNA in SLEDAl was excluded 


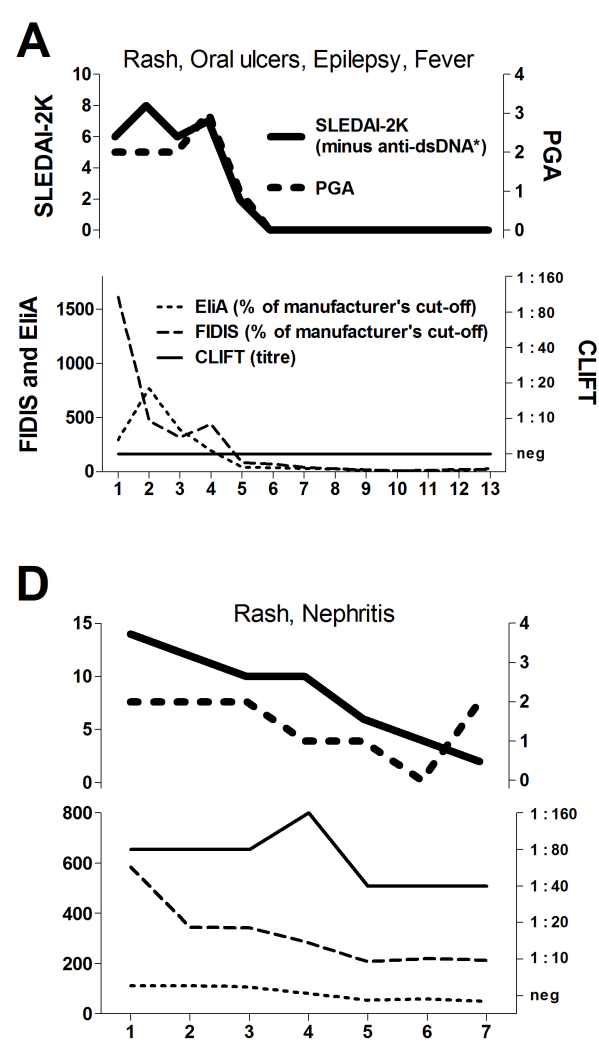

G

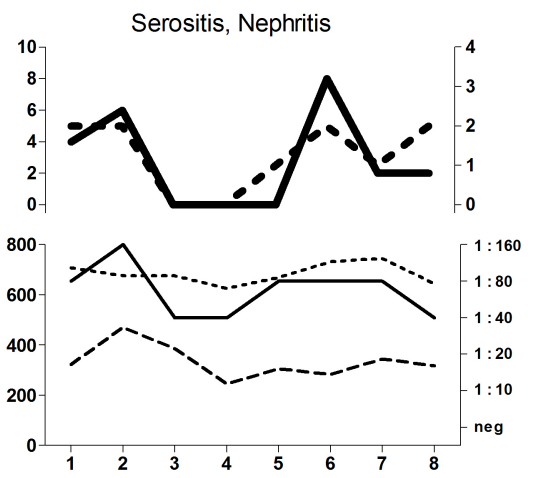

B

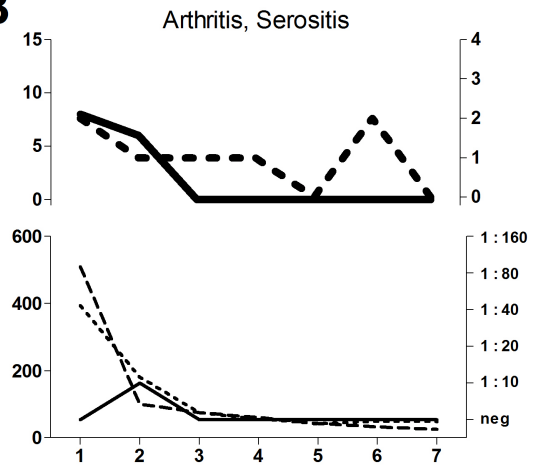

E

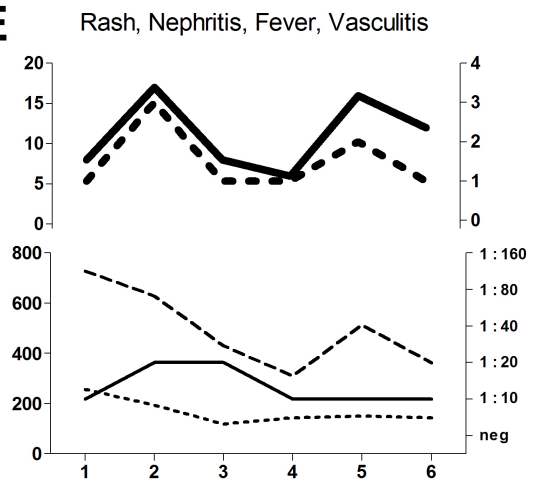

C
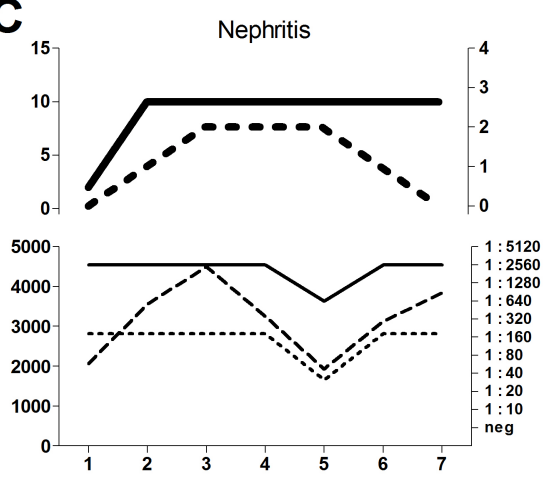

F
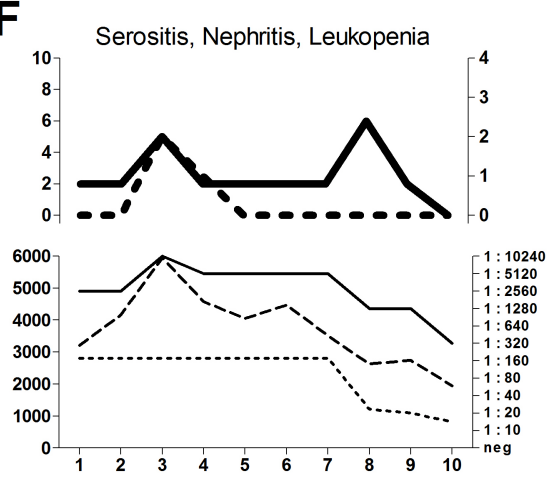

H

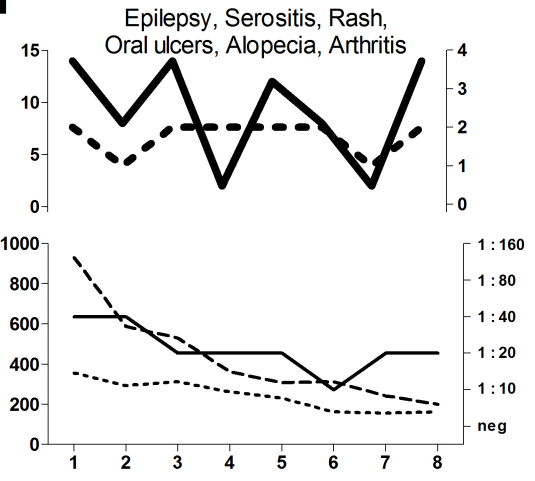

I

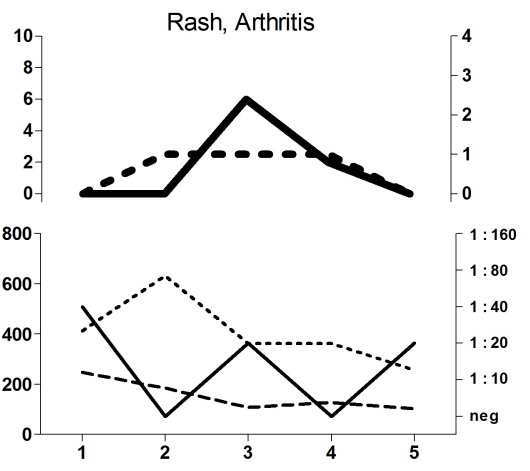


Table 1. Association of SLE disease criteria and anti-dsDNA assessed by four different assays

\begin{tabular}{|c|c|c|c|c|c|c|c|c|}
\hline \multirow[b]{2}{*}{$\begin{array}{l}\text { Cumulative ACR-82 criteria }{ }^{a} \\
\text { (\% fulfilling the criterion) }\end{array}$} & \multicolumn{2}{|r|}{ FIDIS } & \multicolumn{2}{|r|}{ EliA } & \multicolumn{2}{|r|}{ CLIFT } & \multicolumn{2}{|c|}{ EUROLINE } \\
\hline & $p$-value & OR $(95 \% \mathrm{Cl})$ & $p$-value & OR $(95 \% \mathrm{Cl})$ & $p$-value & OR $(95 \% \mathrm{Cl})$ & $p$-value & OR $(95 \% \mathrm{Cl})$ \\
\hline 1. Malar rash (45\%) & 0.325 & $1.41(0.74,2.69)$ & 0.753 & $1.12(0.60,2.08)$ & 0.726 & $0.84(0.42,1.70)$ & 0.870 & $1.08(0.57,2.05)$ \\
\hline 2. Discoid rash (17\%) & 0.084 & $0.40(0.14,1.10)$ & 0.302 & $0.60(0.25,1.43)$ & 0.356 & $0.55(0.20,1.54)$ & 0.391 & $0.62(0.25,1.54)$ \\
\hline 3. Photosensitivity (56\%) & 0.047 & $0.49(0.26,0.95)$ & 0.752 & $0.86(0.46,1.60)$ & 0.003 & $0.33(0.16,0.67)$ & 0.104 & $0.58(0.31,1.11)$ \\
\hline 4. Oral ulcers (10\%) & 0.587 & $1.32(0.46,3.79)$ & 0.035 & $3.00(1.08,8.31)$ & 0.249 & $1.83(0.63,5.28)$ & 0.405 & $1.70(0.61,4.73)$ \\
\hline 5. Arthritis (78\%) & 0.692 & $0.81(0.38,1.73)$ & 0.447 & $0.71(0.34,1.47)$ & 0.674 & $1.30(0.55,3.10)$ & 1.0 & $0.97(0.45,2.11)$ \\
\hline 6. Serositis (38\%) & 0.315 & $1.42(0.73,2.72)$ & 0.746 & $0.87(0.46,1.65)$ & 0.588 & $1.26(0.63,2.55)$ & 0.737 & $0.86(0.44,1.67)$ \\
\hline 7. Renal disorder (21\%) & 0.01 & $2.70(1.29,5.68)$ & 0.004 & $2.98(1.43,6.23)$ & 0.054 & $2.23(1.03,4.85)$ & 0.171 & $1.69(0.80,3.57)$ \\
\hline 8. Neurologic disorder (6\%) & 1.0 & $0.98(0.25,3.98)$ & 0.321 & $1.95(0.54,7.00)$ & 1.0 & $0.77(0.15,3.79)$ & 0.174 & $0.41(0.11,1.49)$ \\
\hline 9. Hematologic disorder (57\%) & 0.013 & $2.42(1.21,4.83)$ & 0.525 & $1.28(0.69,2.41)$ & 0.480 & $1.35(0.67,2.73)$ & 0.621 & $1.23(0.65,2.35)$ \\
\hline 1,2 or 3. Skin $(75 \%)$ & 0.086 & $0.51(0.25,1.04)$ & 1.0 & $1.04(0.51,2.14)$ & 0.001 & $0.29(0.14,0.60)$ & 0.091 & $0.53(0.26,1.08)$ \\
\hline
\end{tabular}

The analysis (Fisher's exact test) is not adjusted for multiple comparisons. 
Table 2. Spearman's correlation between disease variables and anti-dsDNA antibody levels assessed by four assays

\begin{tabular}{|c|c|c|c|c|c|c|c|c|}
\hline \multirow[t]{2}{*}{ Variables } & \multicolumn{2}{|c|}{ FIDIS } & \multicolumn{2}{|c|}{ EliA } & \multicolumn{2}{|c|}{ CLIFT } & \multicolumn{2}{|c|}{ EUROLINE } \\
\hline & rho & p-value & rho & p-value & rho & p-value & rho & p-value \\
\hline $\begin{array}{l}\text { Classical complement } \\
\text { function ( } n=169 \text { ) }\end{array}$ & -0.552 & $<0.0005$ & -0.426 & $<0.0005$ & -0.333 & $<0.0005$ & -0.195 & 0.011 \\
\hline C4 (n=177) & -0.495 & $<0.0005$ & -0.362 & $<0.0005$ & -0.284 & $<0.0005$ & -0.209 & 0.005 \\
\hline C3 (n=178) & -0.371 & $<0.0005$ & -0.251 & 0.001 & -0.218 & 0.003 & -0.154 & 0.040 \\
\hline IFN $\alpha(n=178)$ & 0.323 & $<0.0005$ & 0.269 & $<0.0005$ & 0.215 & 0.004 & 0.137 & 0.068 \\
\hline $\operatorname{ESR}(n=178)$ & 0.193 & 0.010 & 0.176 & 0.019 & 0.112 & 0.135 & 0.081 & 0.280 \\
\hline SLEDAI- $2 K^{\mathrm{a}}(\mathrm{n}=178)$ & 0.148 & 0.048 & 0.121 & 0.109 & 0.096 & 0.203 & 0.034 & 0.657 \\
\hline Physician's global & 0.109 & 0.148 & 0.160 & 0.033 & -0.001 & 0.984 & 0.012 & 0.876 \\
\hline assessment $(n=178)$ & & & & & & & & \\
\hline
\end{tabular}

altem for anti-dsDNA (by CLIFT) excluded.

Variables are organized according to correlation coefficient. 